\title{
CONTRIBUTION A L'ÉTUDE DU SYSTÈME NERVEUX DES FORMES ADULTES ET LARVAIRES DE SCHISTOSOMA MANSONI SAMBON, 1907 (TREMATODA DIGENEA).
}

\author{
II - Rôle de la sérotonine et de la dopamine \\ par E. DEI-CAS*, N. DHAINAUT-COURTOIS** et J. BIGUET*.
}

RÉSUMÉ. Cette étude précise la localisation et le rôle de la sérotonine (5-HT) et de la dopamine (DA) chez Schistosoma mansoni.

Avec les protocoles utilisés,

1) la sérotonine tritiée $\left(5-\mathrm{HT}-{ }^{3} \mathrm{H}\right)$ est incorporée sélectivement par les fibres nerveuses contenant des granules denses mesurant $820 \pm 20 \AA$ (granules de type 2) parfois associées à d'autres structures; la présence de dopamine " froide " dans le milieu ne modifie pas l'incorporation;

2) la dopamine tritiée $\left(\mathrm{DA}^{-}{ }^{3} \mathrm{H}\right)$ est aussi incorporée in vitro par les fibres nerveuses et occasionnellement par les fibres musculaires de Schistosoma mansoni adulte ;

3) le miracidium intraovulaire, les sporocystes de première et de deuxième génération ainsi que les cercaires intrasporocystiques ne semblent pas incorporer la $5-\mathrm{HT}-{ }^{3} \mathrm{H}$ et la $\mathrm{DA}^{-3} \mathrm{H}$. Par contre, les deux amines sont incorporées par l'hépatopancréas de Biomphalaria glabrata et la 5 -HT- ${ }^{3} \mathrm{H}$ a donné un marquage sur certaines zones neuromusculaires du tentacule.

Contribution to the study of nervous system in larval and adult forms of Schistosoma mansoni Sambon, 1907 (Trematoda Digenea).

\section{Role of serotonine and dopamine}

SUMMARY. This study provides details concerning the localization and role of 5-HT and DA in Schistosoma :

1) ${ }^{3} \mathrm{H}-5 \mathrm{HT}$ is incorporated selectively by the nerve fibers containing dense granules measuring $820 \pm 20 \AA$ (type 2 granules), combined at times with other structures; the presence of " cold" dopamine in the environment does not alter the incorporation ;

2) ${ }^{3} \mathrm{H}-\mathrm{DA}$ is also incorporated in vitro by the nerve fibers and occasionally by the muscle fibers of the adult Schistosoma;

3) neither the intraovular miracidium, the first and second generation sporocystes, nor the intrasporocystic cercariae appear to incorporate the ${ }^{3} \mathrm{H}-5-\mathrm{HT}$ or the ${ }^{3} \mathrm{H}-\mathrm{DA}$, under our working condi tions. The two amines are, however, incorporated by the hepatopancreas of Biomphalaria glabrata and the ${ }^{3} \mathrm{H}-5-\mathrm{HT}$ produced marking on certain neuromuscular zones of the tentacle.

* Unité 42 de l'INSERM : Domaine du CERTIA, 369, rue Jules Guesde, 59650 Villeneuve d'Ascq, France.

** Laboratoire de Biologie animale Associé au CNRS, Université de Lille I, 59655 Villeneuve d'Ascq, France. 


\section{Introduction}

Nous avons précisé antérieurement quelques aspects morphologiques du système nerveux de Schistosoma mansoni adulte (Dei-Cas et al., 1980) et abordé l'étude des neuromédiateurs (Dei-Cas et al., 1979). La constitution essentiellement fibreuse des ganglions nerveux, l'absence apparente d'éléments gliaux, l'inexistence d'une gaine séparant le tissu nerveux du parenchyme, la présence de neurones apparemment dispersés dans le parenchyme loin des organes nerveux centraux, et l'existence, à l'intérieur des neurites, au niveau infrastructural, de quatre sortes d'éléments granulaires et vésiculaires à morphologie caractéristique et à taille assez constante ont été décrits. Nos résultats confirment pour l'essentiel l'acquis d'autres auteurs concernant la morphologie du système nerveux des Schistosomes (Loos, 1895; Silk et Spence, 1969 ; Reissig, 1970).

En complément de nos travaux, nous avons localisé par radioautographie la sérotonine (5-HT) dans des neurites contenant surtout des granules denses de type 2 (Dei-Cas et al., 1979). L'incorporation de la $5-\mathrm{HT}-{ }^{3} \mathrm{H}$ ne semble pas être modifiée par la préincubation des Vers dans la dopamine (DA) " froide ». De plus, l'incubation des vers dans la $\mathrm{DA}-{ }^{3} \mathrm{H}$ provoque son incorporation par des neurites qui ne contiennent pas de granules de type 2, mais, parfois, des vésicules synaptoïdes.

Dans la présente contribution, nous nous sommes proposés de confirmer les résultats de nos expériences radioautographiques sur la localisation ultrastructurale de la sérotonine et de la dopamine tritiées et de compléter ce travail en appliquant la même méthodologie aux stades larvaires de Schistosoma.

\section{Matériel et méthodes}

Des adultes, des œufs et des sporocystes de première et de deuxième générations de Schistosoma mansoni (Souche de Surinam, I.N.S.E.R.M. Unité 42 Flers, France) ont été incubés in vitro dans un milieu primaire constitué de sérum de cheval dilué à $75 \%$ dans de l'eau distillée stérile auquel nous avons ajouté les neuromédiateurs " froids" ou marqués au tritium.

Les adultes, prélevés par perfusion après sacrifice de l'hôte par décérébration, sont rincés dans du sérum de cheval à $75 \%$ et préincubés 15 minutes à $370 \mathrm{C}$.

Pour étudier l'incorporation des neuromédiateurs dans les œufs, nous avons traité des fragments de 1 à $3 \mathrm{~mm}^{3}$ d'intestin de hamster parasité de la même manière que les vers adultes.

Les sporocystes, provenant du pied, des tentacules et de la glande digestive de Biomphalaria glabrata, ont été disséqués dans du sérum de cheval dilué à $75 \%$, rincés et préincubés en suivant le même protocole.

Toutes les pièces ont été post-incubées 20 minutes à $370 \mathrm{C}$ dans du sérum de cheval à $75 \%$ " froid " renouvelé 2 à 3 fois, fixés au glutaraldéhyde à $3 \%$ en tampon 
phosphate $0,2 \mathrm{M}, \mathrm{pH} 7,2$ à $4^{\circ} \mathrm{C}$, postfixées au tétroxyde d'osmium, déshydratées à l'acétone et incluses dans l'araldite.

Les coupes semi-fines et ultrafines sont effectuées au microtome LKB, puis soumises à la technique radioautographique (Larra et Droz, 1970 modifiée par Dhainaut-Courtois et Dhainaut, 1976), la durée d'exposition étant de 4 à 8 semaines.

Le détail des expériences est le suivant :

\section{Adultes}

- 5-HT- ${ }^{3} \mathrm{H}^{*}$ (activité spécifique $13,8 \mathrm{Ci} / \mathrm{ml}$ ).

Les vers ont été incubés 10,20 et 60 minutes dans le milieu contenant 40,60 et 100 $\mu \mathrm{Ci} / \mathrm{ml}$ de $5-\mathrm{HT}^{-3} \mathrm{H}$ à $37^{\circ} \mathrm{C}$.

- DA " froide »** et 5 - $\mathrm{HT}-{ }^{3} \mathrm{H}$.

La deuxième fraction d'adultes a été incubée d'abord pendant 60 minutes à $37{ }^{\circ} \mathrm{C}$ dans le milieu contenant $0,005 \mathrm{mg} / \mathrm{ml}$ de dopamine " froide ». Les vers ont été ensuite incubés 60 minutes à $37^{\circ} \mathrm{C}$ dans le milieu contenant $60 \mu \mathrm{Ci} / \mathrm{ml}$ de $5-\mathrm{HT}-{ }^{3} \mathrm{H}$.

- DA- ${ }^{3} \mathrm{H}^{* * *}$ (activité spécifique $2 \mathrm{Ci} / \mathrm{ml}$ )

Les vers ont été incubés pendant 60 minutes à $37^{\circ} \mathrm{C}$ dans le milieu contenant $40 \mu \mathrm{Ci}$ / $\mathrm{ml}$ de $\mathrm{DA}-{ }^{3} \mathrm{H}$.

\section{SPOROCYSTES}

Les sporocystes de première génération ont seulement été incubés dans le milieu contenant la $5-\mathrm{HT}-{ }^{3} \mathrm{H}$ en suivant le même protocole que pour les adultes. Les sporocystes de deuxième génération ont subi les traitements décrits à propos des adultes.

\section{OEuFs}

Les fragments d'intestin de hamster contenant les œufs vivants ont été incubés uniquement dans la $\mathrm{DA}-{ }^{3} \mathrm{H}$ en suivant le protocole décrit pour les adultes.

\section{Résultats}

1. Localisation DE LA $5-\mathrm{HT}^{-3} \mathrm{H}$ ( fig. $I$ et 2 ).

La $5-\mathrm{HT}-{ }^{3} \mathrm{H}$ qui déclenche chez le ver une stimulation très remarquable de la motilité est captée in vitro par le système nerveux central et périphérique de $S$. mansoni.

\footnotetext{
* 5-Hydroxy (G3-H) Tryptamine Sulphate (Code TRK-223) (Batch 49) Radiochemical Centre Amersham.

** Dopamine-Cat. ror 580-I.C.H. Pharmaceuticals, Inc. Life Sciences Group. Cleveland, Ohio, Lote $n^{\circ}{ }_{51} 7 \mathrm{r}$.

*** Dopamine-- ${ }^{3} \mathrm{H}-\mathrm{r}-2$ (Chlorhydrate) C.E.A.
} 

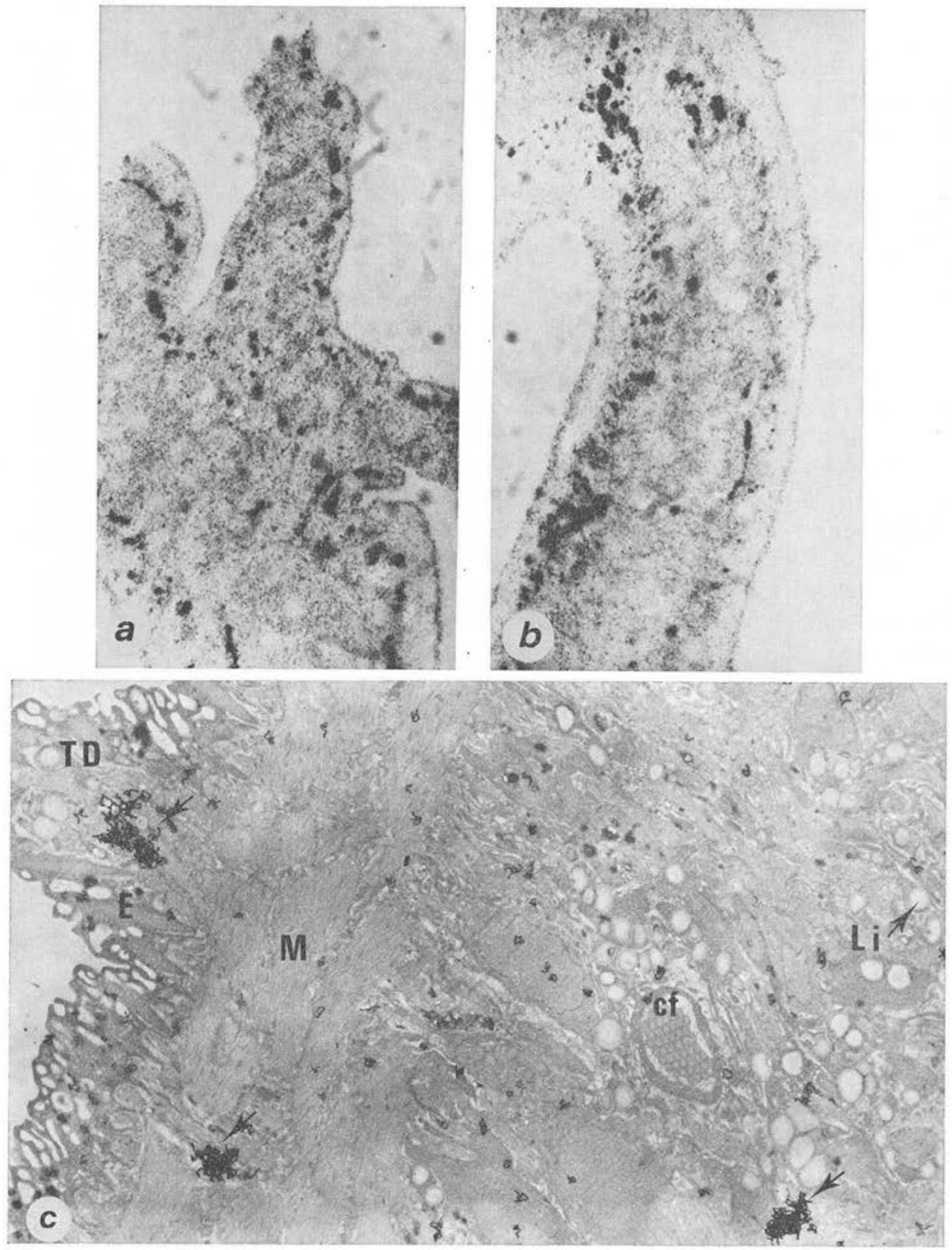

FIG. I. - Localisation de la capture de la 5-HT- ${ }^{3} \mathrm{H}$ (zones noires) par $S$. mansoni adulte. $\mathrm{a}$ et $\mathrm{b}$ : coupes semifines d'un exemplaire mâle.

a : marquage dans la région acétabulaire ;

$\mathrm{b}:$ marquage dans le parenchyme du corps. Incubation dans la $5-\mathrm{HT}-{ }^{3} \mathrm{H}(\mathrm{roo} \mu \mathrm{Ci} / \mathrm{ml}) . \times 800$. c : coupe ultrafine longitudinale : le marquage des régions neuromusculaires est très sélectif (flèches). Incubation dans la $5-\mathrm{HT}-{ }^{3} \mathrm{H}(40 \mu \mathrm{Ci} / \mathrm{ml}) . \times 6000$.

cf : cellule flamme; $\mathrm{E}$ : épine tégumentaire ; Li : lipides; $\mathrm{M}:$ muscle, TD : tubercule dorsal. 

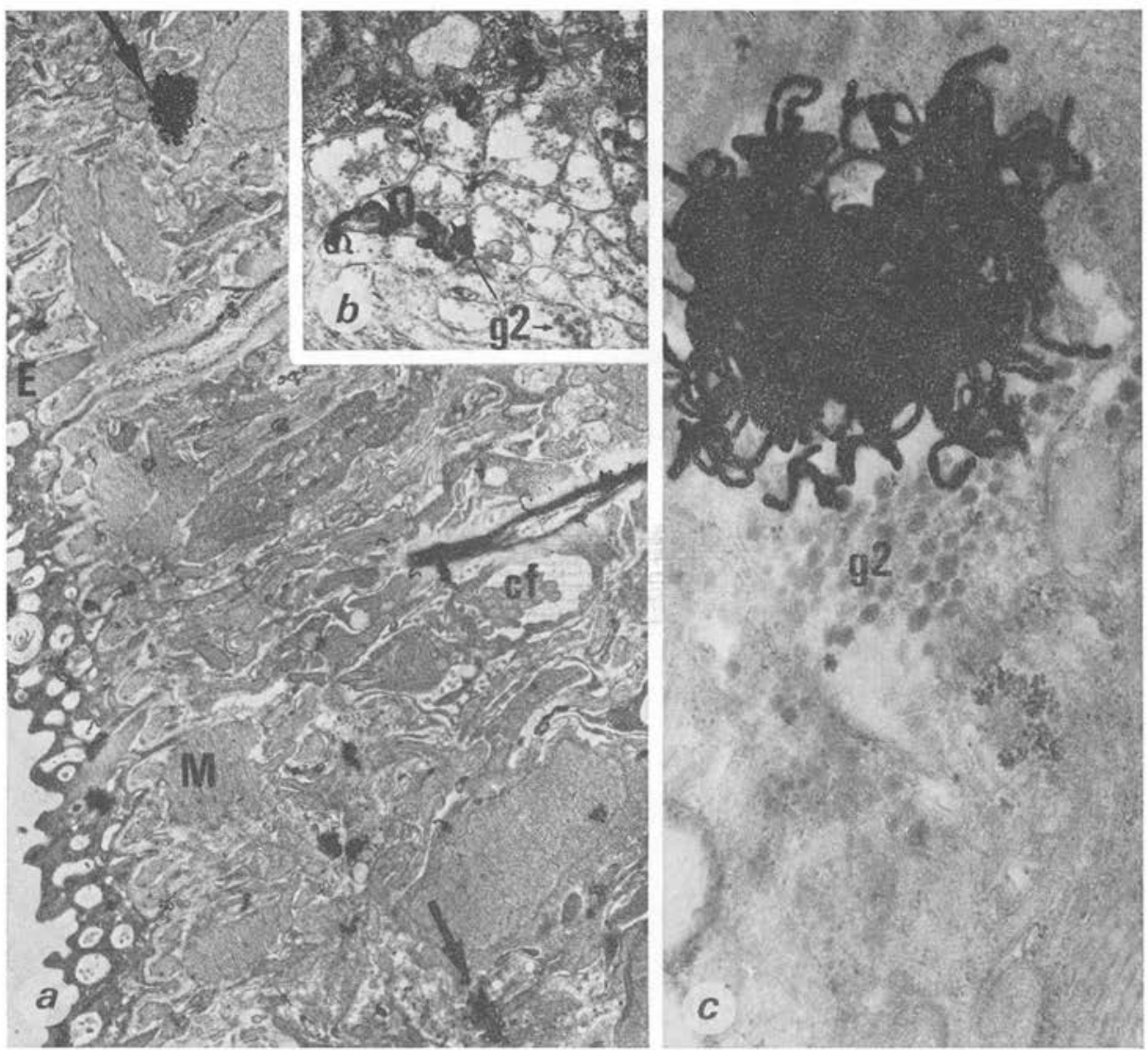

Fig. 2. - Localisation de la capture de la $5-\mathrm{HT}-{ }^{3} \mathrm{H}$ (zones noires) par S. mansoni adulte. Incubation dans la $5-\mathrm{HT}-{ }^{-3} \mathrm{H}(40 \mu \mathrm{Ci} / \mathrm{ml})$.

a : coupe longitudinale d'un mâle : les flèches montrent la localisation très sélective de la 5 -HT- ${ }^{3} \mathrm{H}$ incorporée. Observer l'absence de marquage au niveau des cellules tégumentaires, des fibres musculaires et des cellules flamigères. $\times 8$ ooo.

$\mathrm{b}$ : fibres nerveuses coupées transversalement : capture de la 5 - $\mathrm{HT}-{ }^{3} \mathrm{H}$ après préincubation dans la DA « froide ». $\times 22000$.

c: capture de la 5 - $\mathrm{HT}-{ }^{3} \mathrm{H}$ par une fibre nerveuse contenant des granules de type $2 . \times 43200$. cf : cellule flamme ; E. : épine tégumentaire; g2 : granules de type $2 ; \mathrm{M}$ : muscle.

Sur les coupes semifines non colorées, aussi bien chez le mâle que chez la femelle, il y a une accumulation notable du marquage au niveau des ganglions nerveux. Les fig. Ia et c de notre publication antérieure (Dei-Cas et al., 1979) illustrent efficacement cette affirmation.

Dans le parenchyme sous-tégumentaire, le marquage est disposé de façon assez régulière sur tout le contour du Ver, à distance presque toujours égale de la surface corporelle. Cette distance correspond à l'épaisseur de la paroi du corps (couche tégumentaire et couches musculaires, circulaire et longitudinale). Les zones sous-tégumen- 
taires marquées, sont surtout abondantes dans la région dorsale ( $f i g . r b$ ) de l'animal, mais aussi au niveau de la ventouse orale et de l'acétabulum ( $\mathrm{fig}$. $\mathrm{Ia}$ ). Dans la région rétroacétabulaire, les points de marquage sont plus ostensibles, plus abondants et disposés parfois en rangées.

Les zones de marquage sous-tégumentaires, espacées régulièrement ont été retrouvées en haute résolution ( fig. Ic et 2). Dans ces conditions, la spécificité du marquage est remarquable ; dans les coupes de pièces incubées dans de faibles concentrations de $5-\mathrm{HT}-{ }^{3} \mathrm{H}$, le bruit de fond est en effet presque inexistant.

Occasionnellement, des fibres musculaires isolées ou groupées, situées au niveau de la couche musculaire longitudinale et dans le parenchyme remplissant les tubercules dorsaux du mâle, sont marquées ( $f g . I c$ ). Sur les nerfs coupés, longitudinalement, le marquage suit sensiblement le trajet des fibres. Son association presque constante avec les neurites, surtout lorsque ceux-ci contiennent des granules denses de type 2 $(820 \pm 20 \AA)$, a été confirmée. Par contre, nous n'avons pas réussi à le mettre en évidence dans les péricaryons neuronaux. Ce marquage intéresse très occasionnellement des cellules musculaires. La préincubation dans la DA " froide " ne change pas significativement les résultats.

Les organes sensoriels tégumentaires, les organes digestifs, génitaux et excréteurs, ne sont pas marqués. Il en va de même pour les sporocystes de première et de
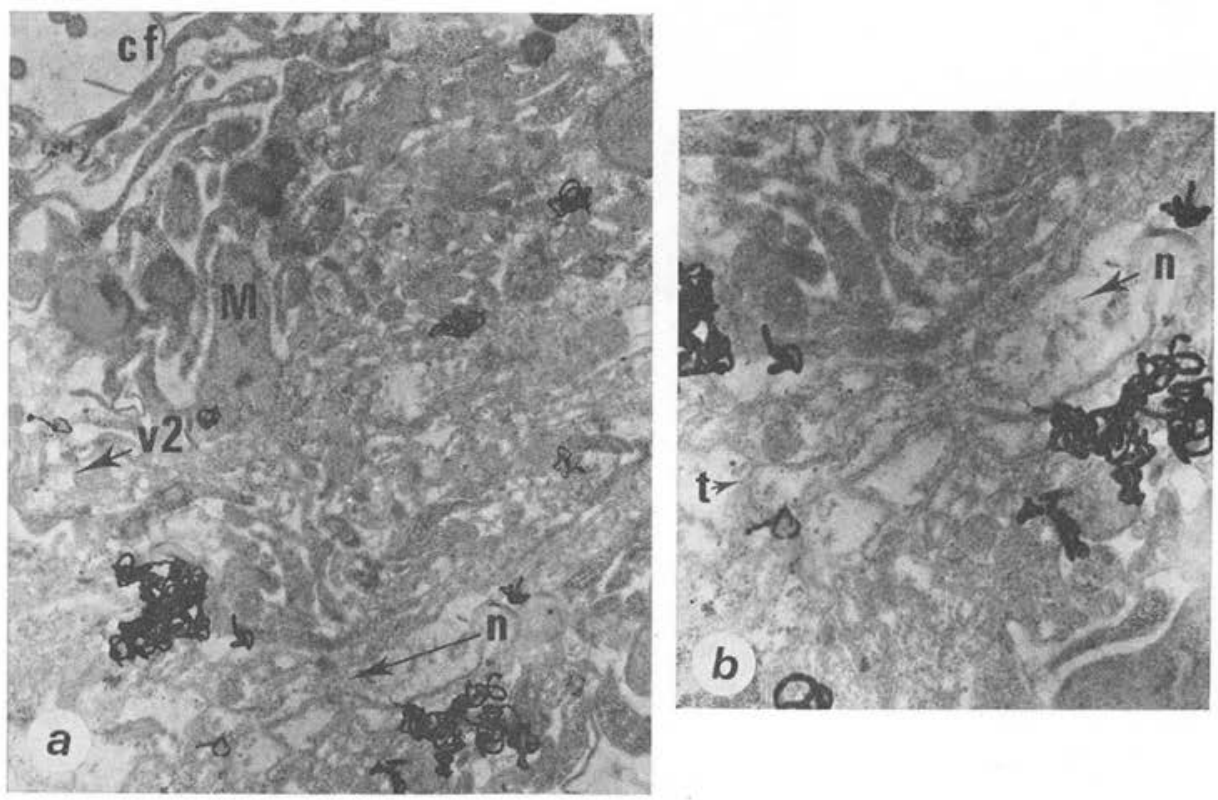

FIG. 3. - Localisation de la capture de la DA- ${ }^{3} \mathrm{H}$ par $S$. mansoni adulte.

a : le marquage est limité aux fibres nerveuses. $\times$ I 4000

b: détail de la fig. a. $\times 32000$;

cf : cellule flamme; $\mathrm{M}$ : muscle; $\mathrm{n}$ : fibres nerveuses; $t$ : microtubules; v2 : vésicules de type 2. 

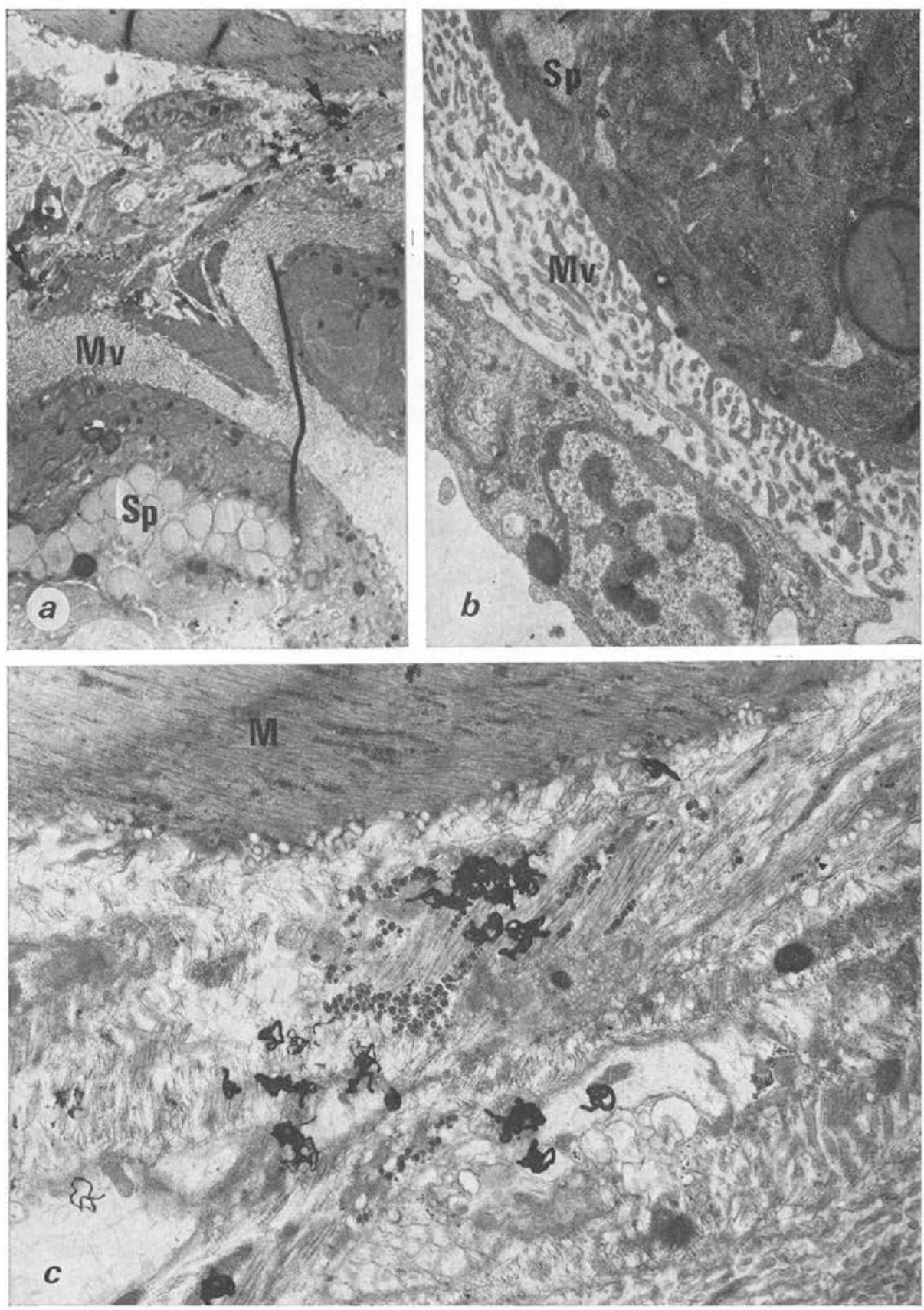

FIg. 4. - Localisation de la capture de la 5-HT- ${ }^{3} \mathrm{H}$ par Biomphalaria glabrata parasité par $S$. mansoni. Fig. a : sporocystes de première génération dans les tissus du tentacule de Biomphalaria. Les microvillosités séparent les sporocystes des tissus du mollusque qui sont marqués (flèche). $\times$ 400o. Fig. $\mathrm{b}:$ vue de l'interphase hôte-parasite. Absence de marquage. $\times 16000$. Fig. c: Détail de la fig. a : capture au niveau d'une zone musculaire du tentacule de Biomphalaria glabrata. $\times$ 1600o. M : muscle; Mv : microvillosités; $\mathrm{Sp}:$ sporocyste. 
deuxième génération alors que l'hépatopancreas et certaines zones neuromusculaires du tentacule des Biomphalaria le sont (fig. 4).

\section{Localisation DE LA DA- ${ }^{3} \mathrm{H}$ (fig. 3).}

Le marquage par la DA- ${ }^{3} \mathrm{H}$ est localisé dans les fibres nerveuses et parfois dans les fibres musculaires. Il n'est malheureusement pas possible de l'associer à une structure neuroplasmique donnée. Les organes digestifs excréteurs, et génitaux ne sont pas marqués. Les sporocystes secondaires et les miracidium intraovulaires ne le sont pas non plus. Par contre, le marquage de certaines cellules de l'hépatopancréas du mollusque et de la sous-muqueuse de la paroi intestinale du hamster est manifeste.

\section{Discussion}

\section{LES AMINES BIOGÈNES CHEZ SCHISTOSOMA ADULTE}

\section{I.I Sérotonine (= 5-HT)}

L'ensemble des résultats histochimiques, biochimiques, pharmacologiques et physiologiques suffit-il pour affirmer le rôle de neuromédiateur activateur de la motilité de la 5-HT chez S. mansoni adulte?

Selon Wolleman (1970) qui rappelle les critères proposés par Florey (1960) pour considérer une substance comme neuromédiatrice (activatrice ou inhibitrice) (p. 19), il n'existerait pratiquement aucun médiateur qui les satisfasse tous.

Des 20 critères cités nous en avons retenu 8 qui nous paraissent essentiels et que nous devons confronter avec les données acquises sur la 5-HT chez Schistosoma :

1) Le neuromédiateur éventuel doit être présent sous forme liée sur toute la longueur du neurone.

On peut admettre que chez Schistosoma adulte la 5-HT satisfait à ce premier critère. En effet, en 1971, Bennett et Bueding ont mis en évidence par histofluorescence, la 5-HT dans les structures nerveuses du ver. Sur les photos et la fig. 1 de leur publication, des cellules et des fibres nerveuses présentent la fluorescence jaune caractéristique. La section des trajets nerveux provoque l'accumulation de la substance fluorescente aux deux extrémités coupées. Nos résultats autoradiographiques à haute résolution nous ont permis de préciser la localisation de la 5-HT qui se situe dans des fibres nerveuses contenant des granules denses de type $2(820 \pm 20 \AA)$ et de confirmer sa présence tout au long des trajets nerveux. Cette constatation, en accord avec les travaux effectués en histofluorescence, suggère fortement l'existence d'un transport axonal de l'amine biogène.

Deux considérations critiques peuvent être cependant faites à propos de notre travail. La première est relative aux limites de la technique utilisée : en effet, un marquage sélectif par une substance donnée, incorporée in vitro ne signifie pas obli- 
gatoirement qu'elle ait la même distribution lorsqu'elle est acquise de façon naturelle par le parasite chez son hôte. La deuxième concerne directement nos résultats : nous n'avons pas réussi, pour des motifs qui nous échappent, à obtenir de marquage dans les péricaryons.

2) Le neurone doit disposer de systèmes enzymatiques lui permettant de synthétiser de novo le neuromédiateur.

Ce critère parait discutable lorsqu'il est appliqué aux parasites obligatoires qui peuvent perdre un certain nombre de voies anaboliques au cours de leur adaptation au parasitisme après avoir acquis la capacité d'incorporer directement à partir du milieu intérieur de l'hôte les substances concernées par ces voies.

Bien que la 5-HT décarboxylase existe peut être encore chez le ver (Bennet et Bueding, 1971), la tryptophane-hydroxylase, essentielle pour la synthèse de la 5-HT ferait défaut (Bennet et Bueding, 1973 ; Bueding et al., 1974). Dans ces conditions le trématode serait incapable de synthétiser de novo la 5-HT qui est par contre incorporée in vitro par le ver (Chou et al., 1973 ; Bennet et Bueding, 1973 : Bueding et al. 1974 ; Senft et al., 1976 ; Dei-Cas et al., 1977 ; Catto et Ottesen, 1979). Aussi Hillman et Senft (1973) suggèrent-ils que la 5-HT et la 5-HTP (= 5-hydroxytryptophane) sont des facteurs nécessairement présents dans le milieu de l'hôte où vit le schistosome. En acceptant ce point de vue qui est très vraisemblable tout se passe comme si le deuxième critère était satisfait.

3) Sous l'effet d'impulsions orthodromiques le neurone doit pouvoir libérer au niveau des terminaisons la substance supposée neuromédiatrice.

Les critères de Florey (1960, cités par Wollemann 1970) concernent ici encore des modèles de métazoaires coelomates où le neurone central nettement isolé des autres tissus est relié par l'intermédiaire d'une synapse à l'organe effecteur. Or, chez les Trématodes, comme chez d'autres Acoelomates, les fibres nerveuses sont noyées dans un parenchyme de structure cellulaire mal connue qui constituerait le vrai milieu intérieur de l'animal.

En fait, les études neurophysiologiques effectuées sur les Schistosomes portent surtout sur la motilité et les variations de la forme du ver sous l'effet de neuromédiateurs, d'anthelmintiques, etc. (Hillman et Senft, 1973, 1975; Hillman et al., 1974; Mason et Fripp, 1976 ; Chavasse et al., 1977, 1979 ; Fetterer et al., 1977, 1978 ; Jewsbury et al., 1977 ; Marshall et al., 1977 ; Pax et al., 1978 ; Kohm et al., 1979). A notre connaissance, on n'a pas encore réussi à stimuler des neurones schistosomiens isolés et on est encore loin de pouvoir le faire. Le troisième critère n'est donc pas satisfait.

Le quatrième et le cinquième critères seront considérés simultanément :

4) Le neurotransmetteur doit pouvoir diffuser dans l'espace synaptique et seule la membrane postsynaptique possédant des récepteurs protéiques spécifiques doit être sensible.

5) L'apport artificiel de faibles concentrations du " neuromédiateur » sur la membrane postsynaptique doit reproduire l'action excitatrice du neurone présynaptique.

L'augmentation de la motilité in vitro du ver en présence de concentrations faibles de 5-HT est un argument en faveur de la bonne diffusion de cette substance 
dans l'espace synaptique et de l'existence de récepteurs spécifiques pour la 5-HT sur la membrane postsynaptique. Mais on pourrait également expliquer cette augmentation de la motilité par une action directe de la 5-HT sur le métabolisme énergétique (Hillman et Senft 1973), car la sérotonine stimule l'adényl-cyclase (Higashi et al., 1973, cités par Catto et Ottesen, 1979) de S. mansoni adulte.

Cependant, nos résultats autoradiographiques, en particulier l'absence de marquage dans des structures non nerveuses, suggèrent bien que le rôle primordial de la 5-HT chez S. mansoni adulte est surtout lié au caractère de neuromédiateur de cette amine biogène. En définitive, il semble raisonnable de considérer que les critères 4 et 5 sont satisfaits.

6) Il doit exister un système inactivant pour le neuromédiateur.

On objective bien la mono-amine-oxydase (M.A.O.) chez Schistosoma (NimmoSmith et Raison, 1968). Cependant, comme les inhibiteurs de la M.A.O. ne modifient pas la motilité ou la teneur en 5-HT des Vers (Bennett et Bueding, 1973), l'enzyme ne constituerait pas le mécanisme principal inactivant de la 5-HT. On peut accepter l'hypothèse que ce mécanisme dépende de la réincorporation active de l'amine par la terminaison nerveuse. L'incorporation de la 5-HT par Schistosoma étudiée par Bennett et Bueding (1973) chez l'adulte et par Catto et Ottesen (1979) chez le schistosomule aurait alors une double signification : apporter au ver un neuromédiateur qu'il ne peut pas synthétiser et interrompre l'action excitatrice du neuromédiateur.

7) La dénervation doit augmenter la sensibilité de la membrane postsynaptique au neuromédiateur.

A notre connaissance, des études de dénervation n'ont pas encore été faites chez le schistosome.

8) Des drogues favorisant ou inhibant l'action du neurone présynaptique doivent agir dans le même sens sur l'effet du neurotransmetteur hypothétique.

A notre connaissance, ce critère n'a pas non plus été satisfait. En fait, dans le domaine pharmacologique, les acquis remarquables concernant les Schistosoma concernent plutôt les effets in vitro de drogues agissant, soit sur le système cholinergique (Tomosky et al., 1974), soit sur le mécanisme d'incorporation de la 5-HT (Bennett et Bueding, 1973 ; Catto et Ottesen, 1979 ; Pax et al., 1979) soit sur la membrane postsynaptique (récepteurs sérotoninergiques) (Tomosky et al., 1974).

Nous savons que les anticholinergiques produisent une augmentation de la motilité du ver par démasquage de l'action activatrice sérotoninergique, puisque le blocage des récepteurs de la 5-HT empêche cet effet (Tomosky et al., 1974), au même titre d'ailleurs que les inhibiteurs du mécanisme d'incorporation de la 5-HT inhibent directement ou indirectement cette augmentation de motilité induite par la sérotonine (Bennett et Bueding, 1973 ; Catto et Ottesen, 1979 ; Pax et al., 1979). En fait, des recherches sur le neurone présynaptique devraient être entreprises pour pouvoir satisfaire le huitième critère.

Des huit critères retenus comme essentiels pour affirmer avec certitude le rôle de neuromédiateur de la 5-HT chez Schistosoma adulte, nous pouvons donc considérer avec les réserves signalées que seuls les critères $1,2,4,5$ et 6 sont satisfaits.

En conclusion, compte tenu des critères cités par Wolleman (1970), la rigueur 
nous empêche provisoirement d'affirmer le rôle de neuromédiateur de la 5-HT chez $S$. mansoni encore qu'il soit très probable et soit généralement reconnu (Bueding, et al., 1974 ; Catto et Ottesen, 1979).

\section{I.2 Dopamine et cathécolamines.}

La dopamine, indépendamment de son activité propre, présente encore l'intérêt d'être un métabolite intermédiaire important du métabolisme de la norépinéphrine et de l'épinéphrine. Elle existe chez Schistosoma et Fasciola hepatica (Chou et al., 1972 ; Gianutsos et Bennett 1977 ; Bennett et Glanutsos, 1977). La norépinéphrine a été démontrée dans les tissus nerveux de Schistosoma adulte (Bennett et Bueding, 1971 ; Machado et al., 1972) qui l'incorpore in vitro (Bennett et Bueding 1973). Les concentrations de dopamine et de norépinéphrine seraient 2 à 3 fois plus élevées chez la femelle que chez le mâle et elles seraient maximales dans la région céphalique (Gianutsos et Bennett, 1977). Dans nos expériences, la dopamine incorporée in vitro par les vers se localise électivement dans les neurites. Il n'est pas certain d'ailleurs que les schistosomes puissent la synthétiser, encore qu'ils disposent de la dopadécarboxylase ainsi que de la dopa- $\beta$-hydroxylase qui permet la synthèse de la norépinéphrine (Seed et al., 1978).

Bien que la dopamine soit un neurotransmetteur connu chez d'autres invertébrés (Gerschenfeld, 1973 ; Tomosky et al., 1974; Bennett et Gianutsos, 1977) nous ne disposons pas de preuves suffisantes pour pouvoir affirmer qu'elle joue ce rôle chez Schistosoma. Elle détermine un allongement corporel du ver adulte in vitro selon Tomosky et al. (1974). Ces auteurs ont apporté des arguments pharmacologiques sur la nature dopaminergique des récepteurs qui participent à ce phénomène. Pour Bennett et Gianutsos (1977) la DA serait chez Fasciola hepatica immature un neurotransmetteur sensoriel et, en même temps, un inhibiteur des synapses qui agirait dans la région céphalique et dans les bords antérieurs du ver.

Selon Bennett et Bueding (1971) les CA pourraient agir comme neurotransmetteurs interneuronaux en provoquant la libération de la 5-HT à partir des sites de stockage, mais il n'est pas exclu que la DA ait un rôle extranerveux. Cependant cette dernière éventualité semble peu probable chez Schistosoma puisque dans nos conditions d'expérimentation nous n'avons pas observé le marquage par la $\mathrm{DA}-{ }^{3} \mathrm{H}$ des organes non neuromusculaires. Pourtant, la découverte chez la femelle de S. mansoni d'une activité phénol-oxydasique (Seed et al., 1978) dont les deux substrats principaux sont la L-Dopa et la dopamine, rend très probable que le dernier ait aussi un rôle dans la reproduction. L'absence de marquage dans les organes génitaux pourrait résulter de la perte de la $\mathrm{DA}^{-}{ }^{3} \mathrm{H}$ en raison de la faiblesse de son affinité pour leurs structures cellulaires, mais notre expérience autoradiographique devrait être répétée après avoir induit l'activité phénol-oxydasique.

\section{2 - AMines BIOGÈnES CHEZ LES STADES LARVAIRES.}

La schistosomule dispose d'un mécanisme d'incorporation de la 5-HT qu'elle pourrait aussi emprunter aux tissus du mollusque au cours du développement larvaire 
(Catto et Ottesen 1979). Cependant dans nos conditions expérimentales, les phases larvaires, miracidiums et sporocystes, n'ont pas incorporé in vitro la 5-HT marquée. Diverses raisons peuvent expliquer cet échec :

Il faut d'abord se souvenir que le succès de la méthode autoradiographique nécessite l'existence d'une capture spécifique de la 5-HT et de la DA, la résistance des deux substances au traitement histologique et la solidité de leur union avec les structures cellulaires. Il a suffi, par exemple, de l'union trop faible de la DA avec les structures cellulaires du cerveau des vertébrés pour rendre difficile son étude autoradiographique au niveau de cet organe (Taxi, 1976).

L'absence de marquage des sporocystes peut encore résulter de la présence de quantités non négligeables de 5-HT "froide " dans les tissus de Biomphalaria glabrata (Chiang $e t a l ., 1974)$. Enfin des obstacles peuvent être apportés à la pénétration des molécules marquées. Ceci est particulièrement vraisemblable en ce qui concerne l'œuf qui possède une coque épaisse et qui, dans nos expériences, se trouvait englobé dans le granulome réactionnel de l'hôte.

Ces considérations inciteraient d'abord à répéter nos manipulations sur des stades larvaires (miracidium, sporocystes, cercaires) isolés. Les mêmes considérations peuvent être faites à propos du défaut de marquage des stades larvaires par la DA tritiée qui est en désaccord avec l'hypothèse de Tomosky et al. (1974) selon laquelle les récepteurs dopaminergiques existeraient déjà au moment du passage chez le mollusque. Dans notre travail, l'hépatopancréas de Biomphalaria glabrata a incorporé a $5-\mathrm{HT}^{-3} \mathrm{H}$ et la $\mathrm{DA}-{ }^{3} \mathrm{H}$ et les régions neuromusculaires du tentacule du mollusque la 5-HT- ${ }^{3} \mathrm{H}$ [les observations de Chiang et al. (1974) se limiteraient aux organes nerveux de $B$. glabrata]. Ces observations ont leur intérêt car il est probable qu'il existe des influences réciproques entre neuromédiateurs de l'hôte et du parasite.

\section{Conclusion}

L'étude autoradiographique du système nerveux de Schistosoma mansoni nous a permis de préciser la localisation de la sérotonine et de la dopamine tritiée incorporées in vitro par le ver adulte et, par ce moyen, d'apporter des arguments biomorphologiques supplémentaires en faveur du rôle joué par ces substances dans la neurotransmission chez le ver. Leur distribution spécifique dans le tissu nerveux du Trématode appuie cette hypothèse par ailleurs largement acceptée par plusieurs auteurs. Cette activité de neuromédiateur n'exclut d'ailleurs pas une fonction extranerveuse des deux amines (stimulation du métabolisme énergétique pour la sérotonine, participation à la formation de la coque ovulaire pour la dopamine).

Le miracidium intraovulaire, les sporocystes de première et deuxième génération ainsi que les cercaires intrasporocystiques, n'ont pas incorporé la sérotonine et la dopamine tritiées, contrairement aux tissus de Biomphalasia glabrata, mais il est possible que nos conditions expérimentales soient la cause de cet échec. 


\section{BIBLIOGRAPHIE}

Bennett J., Bueding E. Localization of biogenic amines in Schistosoma mansoni. Comp. Biochem. Physiol. 1971. 39 A, 857-867.

Bennett J. L., Bueding E. Uptake of 5-hydroxytryptamine by Schistosoma mansoni. Molec. Pharmacol., 1973, 9, 311-319.

Bennett J., Bueding E., Timms A. R., Engstrom R. G. Occurrence and levels of 5-hydrocytryptamine in Schistosoma mansoni. Molec. Pharmacol. 1969, 5, 542-545.

Bennett J. L., Gianutsos G. Distribution of cathecolamines in immature Fasciola hepatica : a histochemical and biochemical study. Int. J. Parasitol., 1977, 7, 221-225.

Bueding E., Bennett H. L., Chou T., Pert C., Tomosky T. Effect of antischistosomal drugs on the uptake of 5 -hydroxytryptamine by Schistosoma mansoni. Proc. ${ }^{2}$ rd Int. Congr. Parasitol., 1974, 3, I443-1 444 .

Catto B. A., Ottesen E. A. Serotonin uptake in Schistosomules of Schistosoma mansoni. Comp. Biochem. Physiol., 1979, 63 C, 235-242.

Chavasse C. J., Brown M. C., Norman D., Gilles H. M., Bell D. R. Method for monitoring Schistosome motility in vitro using ultrasound (Technical note). Trans. Roy. Soc. Trop. Med. Hyg., 1977, 7I, I15-116.

Chavasse C. J., Brown M. C., Bell D. R., Schistosoma mansoni : ultrasonically detectable motor activity responses to 5-hydroxytryptamine in vitro. Ann. Trop. Med. Parasitol., 1979, 73 , 363-367.

Chiang P. K., Bourgeois J. G., Bueding E. 5-hydroxytryptamine and dopamine in Biomphalaria glabrata. J. Parasitol. 1974, 60, 264-271.

Chou T. S., Bennett J., Bueding E. Occurrence and concentrations of biogenic amines in trematodes. J. Parasit., 1972, 58, 1098-1102.

Chou T. S., Bennett J., Pert C., Bueding E. Effect of hycanthone and of two of its structural analogs on levels and uptake of 5-hydroxytryptamine in Schistosoma mansoni. J. Pharmacol. Exp. Ther., 1973, $186,408-415$.

Dei-Cas E., Dhainaut Courtois N., Dhainaut A. Étude de la localisation de la sérotonine chez l'adulte de Schistosoma mansoni. Journées de Mycologie et Parasitologie de la France, I I-I2 mai 1977, Angers (France).

Dei-Cas E., Dhainaut-Courtois N., Vernes A. Contribution à l'étude du système nerveux de Schistosoma mansoni Sambon I9o7 (Trématode : Digenea). I. Aspects morphologiques : neuroanatomie, histologie et ultrastructure. Ann. Parasitol. Hum. Comp., 1980, 55, 69-86.

Dei-Cas E., Dhainaut-Courtois N., Dhainaut A., Vernes A. Ultrastructural localization of the $3 \mathrm{H}-5$-HT of adults Schistosoma mansoni. J. Biol. Cell., I979, 35, 32 I-324.

Dhainaut-Courtors N., Dhainaut A. Gamma Aminobutyric Acid and 5-hydroxytryptamine in the nervous system of an Annelid Worm (Nereis diversicolor). J. Microscopie Biol. Cell., $1976,27,26 \mathrm{I}-266$.

Fetrerer R. H., Pax R. A., Bennett J. L. Schistosoma mansoni : Direct method for simultaneous recording of electrical and motor activity. Exp. Parasitol., 1977, 43, 286-294.

Fetterer R. H., Pax R. A., Strand S., Bennett J. L. Schistosoma mansoni physical and chemical factors affecting the mechanical properties of the adult male musculature. Exp. Parasitol., $1978,46,59-71$.

Gerschenfeld H. M. Chemical transmission in invertebrate central nervous system and neuromuscular junctions. Physiol. Rev., I973, 53, I-II9.

Gianutsos G., Bennett J. L. The regional distribution of dopamine and norepinephrine in Schistosoma mansoni and Fasciola hepatica. Comp. Biochem. Physiol., 1977, 58 C, I57-159.

Hillman G. R., Senft A. W. Schistosome motility measurements : response to drugs. J. Pharmacol. Exp. Ther., 1973, $185,177-184$.

Hillman G. R., Olsen N. J., Senft A. W. Effect of methysergide and dihydroergotamine on Schistosoma mansoni. J. Pharm. Exp. Therapeutic., 1974, $188,529-535$. 
Hillmann G. R., Senft A. W. Anticholinergic properties of the antischistosomal drug hycanthone. Am. J. Trop. Med. Hyg., I975, 24, 827-834.

Jewsbury J. M., Homewood C. A., Marshall I. L. Inexpensive apparatus for measuring activity of Schistosoma mansoni in vitro (Technical note). Trans. R. Soc. Trop. Med. Hyg., 1977, 71 , II 5 .

Kohm A., Tendler M., Fernandes B. M. M., Pinto R. M., Castillo E. A. Schistosome activity in saline media. Rev. Brasileira Pesquisas Med. Biol., I979, I2, I23-1 26.

LARRA F., Droz B. Techniques radioautographiques et leurs applications à l'étude du renouvellement des constituants cellulaires. J. Microsc, 1970, 9, 845-88o.

Looss A. Zur Anatomie und Histologie der Bilharzia haematobia (Cobbold). Arch. mikr. Anat.' 1895, 46, I-108.

Machado C. R. S., Machado A. B. M., Pellegrino J. Cathecolamine containing neurons in Schistosoma mansoni. Z. Zellforsch. Mikr. Anat., 1972, 124, 230-237.

Marshall I., Homewood C. A., Jewsbury J. M. Measurement of the activity in vitro of Schistosoma mansoni. Trans. Roy. Soc. Trop. Med. Hyg., 1977, 71, 287.

MASON P. R., FRIPP P. J. Analysis of the movements of Schistosoma mansoni miracidia using dark-ground photography. J. Parasitol., 1976, 62, 721-727.

Nimmo-Smith R. H., Raison C. G. Mono-amine oxydase activity of Schistosoma mansoni. Comp. Biochem. Physiol., 1968, 24, 403-416.

Pax R., Bennett J. L., Fetterer R. A benzodiazepine derivative and praziquantel : effects on musculature of Schistosoma mansoni and Schistosoma japonicum. Arch. Pharmacol., 1978, $304,309-315$.

Pax R., Fetterer R., Bennetr J. L. Effects of fluoxetine and imipramine on male Schistosoma mansoni. Comp. Biochem. Physiol., 1979, 64 C, 123-127.

ReIssig M. Characterization of cell types in the parenchyma of Schistosoma mansoni. Parasitol., I970, 60, 273-279.

Seed J. L., Boff M., Bennetr J. L., Phenol oxydase activity : induction in female schistosome by in vitro incubation. J. Parasit., 1978, 64, 283-289.

Senft A. W., Senft G. R., Hillman D. P., Kryger S. Influence of hycanthone on morphology and serotin uptake of Schistosoma mansoni. Am. J. Trop. Med. Hyg., 1976, 25, 832-840.

Silk M. H., Spence I. M. : Ultrastructural studies of the blood fluke Schistosoma mansoni III. The nerve tissue and sensory structures. S. Afr. J. Med. Sci., 1969, 34, 93-104.

TAXI J. General principles of neurotransmitter detection. Problems and application to catecholamines. J. Microsc. Biol. Cell., 1976, 27, 243-248.

Tomosky T. K., Bennett J. L., Bueding E. Tryptaminergic and dopaminergic responses of Schistosoma mansoni. J. Pharmacol. Exp. Ther., 1974, 190, 206-271.

Wolleman M. Métabolisme des médiateurs chimiques du système nerveux à l'état physiologique et pathologique. Masson, Paris, 1970. 